\title{
A Comparison of Robustness and Performance of Linear and Non linear Lanchester Dampers
}

\author{
Mohammad Vakilinejad, Aurélien Grolet, Olivier Thomas \\ Arts et Métiers ParisTech, \\ Laboratoire d'Ingénierie des Systèmes Physiques et Numériques \\ (LISPEN EA 7515) \\ 8 boulevard Louis XIV 59046 Lille, France \\ aurelien.grolet@ensam.eu
}

\section{abstract}

In this paper, we study and compare performance and robustness of linear and non linear Lanchester dampers. The linear Lanchester damper consists of a small mass attached to a primary system through a linear dashpot, whereas the non linear Lanchester damper is linked to the primary mass through dry friction forces. In each case, we propose a semi-analytical method for computing the frequency response, for different values of the design parameters, in order to evaluate the performance and robustness of the two kinds of damper. Overall, it is shown that linear Lanchester dampers perform better than non linear damper both in terms of efficiency and robustness. Moreover, the non linear frequency response curves, that include the intrinsic non-smooth nature of the friction force, may serve as reference curve for further numerical studies.

\section{Introduction}

In the engineering and industrial world, a lot of systems are subjected to oscillatory forces which in turn give birth to vibrations. Most of the time those vibrations are an inconvenience and one often wants to reduce the amplitude of vibration to a minimum. Several strategies (active or passive) can be used to suppress or at least reduce the vibration amplitude.

Active methods contain sensors, actuators and control units and require energy sources. These methods possess excellent properties and efficiencies, but involve complexities in the design of sensors and actuators (piezoelectric, electromagnetic, hydraulic...) as well as in the controllers [1], require energy supply and may be unstable, which may not be suitable for many applications. Passive or semi passive techniques such as electromechanical shunts share the same features with the advantage of being intrinsically passive most of the time $[2,3,4]$.

Passive methods, on the other hand, are composed of basic mechanical elements. They are designed to affect the vibration behaviour of a system by either changing the key overall 
structural properties such as resonance / antiresonance frequencies or by adding auxiliary energy dissipation elements. Viscoelastic elements [5, 6], Tuned Mass Dampers [7] and Lanchester dampers [8] are among the most known passive methods.

Tuned mass damper have a wide range of application. They consist of a small mass being attached to the primary system through well designed stiffness and viscous damper. Several tuning strategies are at hand $([9,7])$ to find optimal values for the stiffness and damping element of the absorber. For rotating machineries, analogous devices such as pendulum absorbers tuned on a particular excitation frequency can also be used $[10,11]$. Tuned mass damper performs well at the design point but are not very robust, and linear lanchester damper appears to be superior with relation to robustness. The same results hold for their electromechanical analogs [3, 12].

A linear Lanchester damper is simply a tuned mass damper without the stiffness, i.e. it consists of a small mass linked to the primary structure only through a viscous dashpot [13]. In application, it is usually used for rotating systems (e.g. thermal engines), where it consists of a flywheel, generally shaped as a ring, free to rotate within a casing filled with a fluid with high viscosity, for example, a silicon-based oil [14]. However, due to inconveniences regarding maintenance and sealing, another type of damper, which has been proposed by Lanchester in [15], is used as an alternative in many applications. The latter uses dry friction forces, instead of viscous effects, to dissipate energy and thus reduce vibration levels.

The use of friction force to reduce vibration level is well established, for example, in turbo machinery, where compressor or turbine blades are linked to the disk through dovetail joins which generates friction [16]. In the case of blisks (monobloc bladed disk), a friction ring can be used to reduce the vibration amplitude [17]. In the remaining of the paper, the Lanchester damper with viscous effect will be referred to as a linear Lanchester damper, and the absorber based on dry friction force will be referred to as a non linear Lanchester damper, due to the non linear non-smooth nature of the coulomb friction.

Historically, the case of dry friction seems to have been studied before the case of linear viscous damping. Its effect on the motion of a single degree of freedom system has been studied in 1931 by Den Hartog [18]. Some years before, the same author considered non linear Lanchester dampers including a dry friction element, with a fairly thorough work [19]. Due to the lack of modern computation and sensor devices, the authors had to involve simplifying assumptions and their contribution and results were pioneering for long. Their simplifying assumptions include constant phase difference of 90 degrees between the input torque and the primary system displacement, no counter effect of the damper-flywheels on the motion profile in the steady state situation and more importantly, lack of damping effect in the primary system. More recently, the results presented in [19] were reconsidered by removing assumptions over the phase difference between the input torque and system displacement and pure sinusoidal motion profile for the primary system by Ye in [20]. In their work, they showed that for an undamped primary system, the results derived from the energy approach involving all the simplifying assumptions by Den Hartog [19] were close enough to the exact values. In parallel, the case of the Lanchester damper with a linear viscous element, more simple because of its pure linear transfer function, has been considered and optimized by several works $[13,21,22]$.

In this paper, we aim to compare the two kinds of Lanchester absorbers (linear and non linear). In section 2, a linear Lanchester damper is considered. We first propose an analytical 
approximation of the efficiency, which is used to derive an approximation of the optimal design parameters for the absorber. Then, those results are validated using a numerical approach allowing to find the "exact" optimal value for the design parameters. The section ends by the study of the efficiency and robustness of such a linear Lanchester absorber. Section 3 is dedicated to the study of a non linear Lanchester absorber. We use a semi analytical approach similar to the one proposed in [18] to compute the frequency response of the system. Then the optimal design parameters are obtained through numerical simulation. Finally, the efficiency and robustness of non linear damper are studied. Section 4 is devoted to the comparison between the two kinds of dampers. The paper ends with some concluding remarks.

\section{Linear Lanchester Damper}

In this part, one focuses on a primary structure coupled to a linear Lanchester damper. A single mode approximation is considered for the primary structure so that it can be represented by a single degree of freedom with modal characteristics $M, k$ and $c_{1}$ [23]. In order to simplify the presentation, a translational system is considered here. The overall schematic of a such a system is shown in figure 1 . The characteristics of the absorber device are $m$ and $c_{2}$.

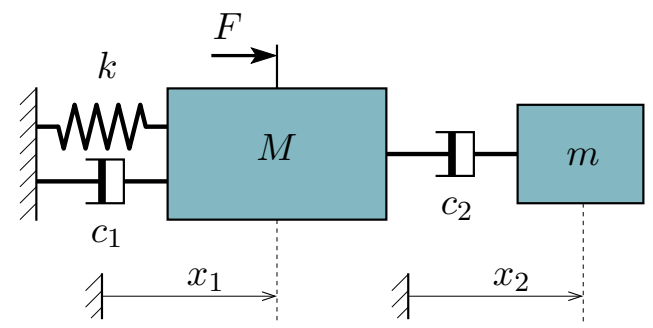

Figure 1: Schematics of a linear Lanchester absorber connected to a primary system of mass $M$

\subsection{Governing Equations}

Using Newton's law, one can derive the set of equations governing the motion of the system in Fig.1, given as:

$$
\begin{aligned}
M \ddot{x_{1}}+c_{1} \dot{x_{1}}+k x_{1}-c_{2}\left(\dot{x_{2}}-\dot{x_{1}}\right)=F(t), \\
m \ddot{x_{2}}-c_{2}\left(\dot{x_{1}}-\dot{x_{2}}\right)=0 .
\end{aligned}
$$

The relative displacement $x_{d}$ between the absorber and the primary structure is defined as :

$$
x_{d}=x_{2}-x_{1} .
$$

Using the relative displacement, equations (1a) and (1b) can be rewritten as:

$$
\begin{aligned}
& (1+\mu) \ddot{x_{1}}+\mu \ddot{x_{d}}+2 \xi \omega_{1} \dot{x_{1}}+\omega_{1}^{2} x_{1}=\frac{F(t)}{M}, \\
& \ddot{x_{1}}+\ddot{x_{d}}+\Lambda \dot{x_{d}}=0,
\end{aligned}
$$


where the new parameters $\omega_{1}, \xi, \Lambda$ and $\mu$ are given by:

$$
\omega_{1}=\sqrt{\frac{k}{M}}, \xi=\frac{c_{1}}{2 \sqrt{k M}}, \Lambda=\frac{c_{2}}{m}, \mu=\frac{m}{M} .
$$

Parameters $\omega_{1}, \xi, \mu$ and $\Lambda$ are respectively the natural frequency and the damping ratio of the primary system, the mass ratio and damping coefficient of the linear Lanchester absorber. Finally, we consider a harmonic excitation $F(t)=F \sin (\Omega t)$, we introduce a non dimensional time $\bar{t}$ and a non-dimensional displacements $\bar{x}_{i}$ defined as follows (for $i=1,2$ ):

$$
\bar{t}=\omega_{1} t, \bar{x}_{i}=\frac{k x_{i}}{F}
$$

Substituting the previous parameters into the equation of motion results in the following non-dimensional form for the equation of motion:

$$
\begin{aligned}
& (1+\mu) \ddot{\bar{x}}_{1}+\mu \ddot{\bar{x}}_{d}+2 \xi \dot{\bar{x}}_{1}+\bar{x}_{1}=\sin (\omega \bar{t}), \\
& \ddot{\bar{x}}_{1}+\ddot{\bar{x}}_{d}+\lambda \dot{\bar{x}}_{d}=0
\end{aligned}
$$

with $\omega$ being the non dimensional excitation frequency and $\lambda$ being the non dimensional damping coefficient of the absorber, defined as follows:

$$
\omega=\frac{\Omega}{\omega_{1}}, \lambda=\frac{\Lambda}{\omega_{1}} .
$$

The non dimensional equations(5a) and (5b) show that there is only three independent design parameters $(\mu, \lambda$ and $\xi)$. Note that, in the following, the bars denoting non dimensional quantity will be dropped to lighten the notations.

Applying Fourier transform to equations (5a) and (5b) results in the Frequency Response Function (FRF) $H$ (between the displacement of the primary structure and the excitation), given as:

$$
H(\omega)=\frac{\hat{x}_{1}(\omega)}{\hat{F}(\omega)}=\frac{\lambda+i \omega}{\lambda-\omega^{2}(2 \xi+\lambda(1+\mu))+i \omega\left(1+2 \xi \lambda-\omega^{2}\right)},
$$

where $\hat{o}(\omega)$ is the Fourier transform of $\circ(t)$ and $i^{2}=-1$.

As an example, the surface representing the modulus $|H(\omega)|$ as a function of the frequency for different damping values of the absorber is shown in figure 2. One can see that for every value of $\lambda$ (damping), there is a corresponding frequency for which $|H|$ is maximum (resonance point). One can also see that the maximum of the FRF have a minimum along the $\lambda$ axis. In other words, there exists an optimal damping ratio $\left(\lambda_{\text {opt }}\right)$ for which the resonance amplitude of the FRF is minimum (among all other possible FRF). This value creates a saddle point on the $|H(\omega)|$ surface.

\subsection{Efficiency of The Linear Lanchester Damper}

Let us start by introducing our efficiency metric. In the remaining of this paper, the efficiency of the dampers will be characterized by a property denoted $A_{d B}$ and called efficiency. This parameter is defined as the ratio of the maximum amplitude of the FRF of a system without 


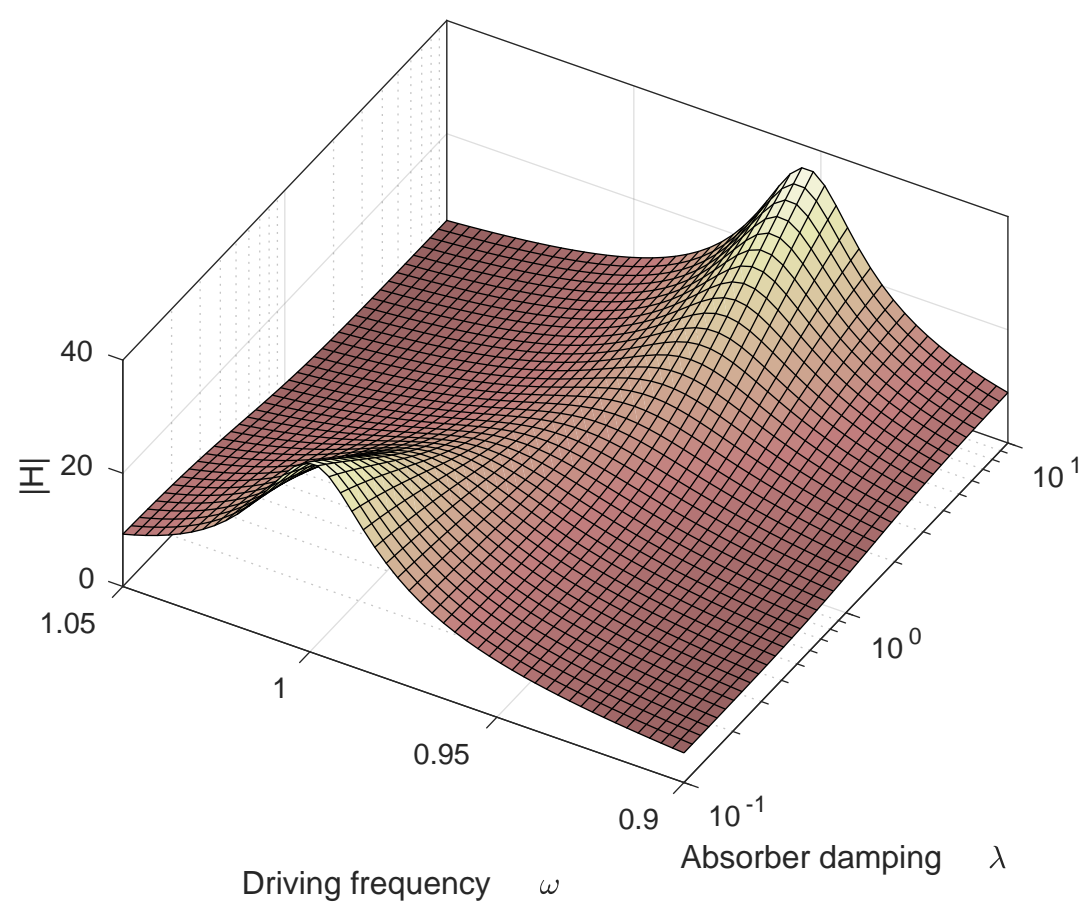

Figure 2: Surface of modulus $|H(\omega)|$ for a primary system with parameters $\xi=0.01$ and $\mu=0.1$

damper $(\lambda=0)$ over the maximum amplitude of the FRF of a system with optimal damper $\left(\lambda=\lambda_{\text {opt }}\right)$. The decibel scale is used so that the expression for $A_{d B}$ is:

$$
A_{d B}=20 \log \left[\frac{\max _{\omega}\left(|H(\omega)|_{\lambda=0}\right)}{\max _{\omega}\left(|H(\omega)|_{\lambda=\lambda_{\text {opt }}}\right)}\right] .
$$

A graphical interpretation of $A_{d B}$ is depicted on the right hand side of figure 3 .

\subsubsection{Analytical estimation of the efficiency $A_{d B}$}

The FRF curves of $x_{1}$ for different values of $\lambda$ are shown in figure 3 . When there is no damping for the primary system $(\xi=0)$, one observes the remarkable property that all the FRF curves go through one particular point (denoted $F$ on figure 3) [13].

To obtain an estimation of $A_{d B}$, the first step is to find the crossing frequency $\omega_{c}$ (corresponding to the abscissa of point $F$ on fig.3). For this, one can use the two FRF related to $\lambda=0$ and $\lambda=\infty$ (absorber stuck to the primary mass), the frequency $\omega_{c}$ being defined as:

$$
\left|H\left(\omega_{c}\right)\right|_{\lambda=0}=\left|H\left(\omega_{c}\right)\right|_{\lambda=\infty}
$$

Using equation (7) and solving the previous equation for $\omega_{c}$ gives:

$$
\omega_{c}=\sqrt{\frac{2}{2+\mu}} .
$$




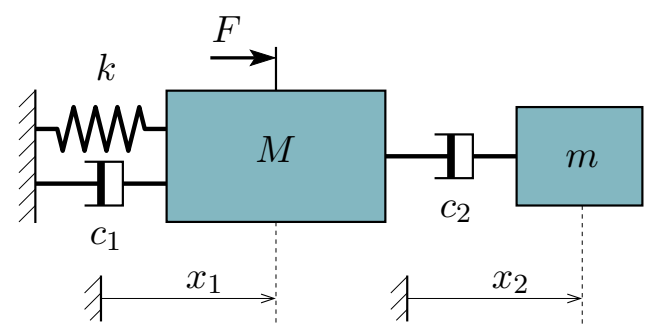

Figure 3: Example of FRF for an undamped primary system (left, $\xi=0$ ) and for a damped primary system (right: $\xi \neq 0$ )

The second step in deriving an expression for $A_{d B}$ is to find the optimal value of $\lambda$. When $\xi=0$, the optimal value of $\lambda$ is the one for which the maximum of the FRF happens for $\omega=\omega_{c}$ (i.e the maximum of the FRF is located exactly at point $F$ ). This can be translated into the following condition:

$$
\left.\frac{\partial|H(\omega)|}{\partial \omega}\right|_{\omega=\omega_{c}}=0 .
$$

Solving the previous equation for $\lambda$ gives an estimation for the optimal damping value (when $\xi=0)$ :

$$
\lambda_{\text {est }}=\sqrt{\frac{2}{(1+\mu)(2+\mu)}} .
$$

Which is in agreement with the results presented in [13] and [21].

To compute $A_{d B}$ in the general case $(\xi \neq 0)$, we will assume that the optimal value of $\lambda$ given in Eq.(12) is still valid even if $\xi \neq 0$. Also we assume that the maximum of the related FRF still happens for $\omega=\omega_{c}$. Thus the term $\max _{\omega}\left(|H(\omega)|_{\lambda=\lambda_{\text {opt }}}\right)$ in equation (8) is approximated by the following:

$$
\max _{\omega}\left(|H(\omega)|_{\lambda=\lambda_{\mathrm{opt}}}\right)=\left|H\left(\omega_{c}\right)\right|_{\lambda=\lambda_{\mathrm{est}}} .
$$

Substituting the results of equations (10) and (12) into Eq.(7), one gets:

$$
\left|H\left(\omega_{c}\right)\right|_{\lambda=\lambda_{\text {est }}}^{2}=\frac{(2+\mu)^{5 / 2}}{8 \sqrt{2} \mu \xi \sqrt{(1+\mu)}+8 \xi^{2}(2+\mu)^{3 / 2}+\mu^{2} \sqrt{2+\mu}} .
$$

When $\lambda=0$, Eq.(7) gives the maximum amplitude of the response of a single degree of freedom with damping and without any additional mass (no absorber):

$$
\max _{\omega}\left(|H(\omega)|_{\lambda=0}^{2}\right)=\frac{1}{4 \xi^{2}\left(1-\xi^{2}\right)} .
$$

Using Eq.(14) and (15) an estimation of the efficiency $A_{d B}$ is finally given by:

$$
A_{d B}=10 \log \frac{8 \sqrt{2} \mu \xi \sqrt{(1+\mu)}+8 \xi^{2}(2+\mu)^{3 / 2}+\mu^{2} \sqrt{2+\mu}}{4 \xi^{2}\left(1-\xi^{2}\right)(2+\mu)^{5 / 2}} .
$$




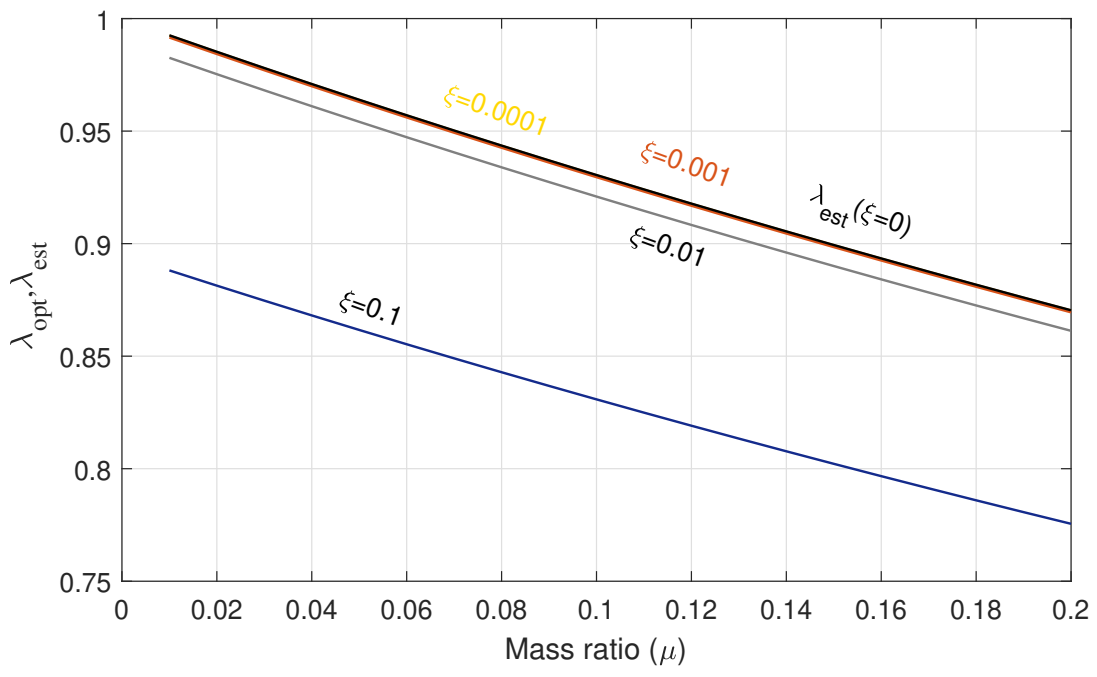

Figure 4: Values of estimated damping ratio $\lambda_{\text {opt }}$ and optimal damping ratio $\lambda_{\text {est }}$ as a function of mass ratio $(\mu)$ for different primary damping ratio $\xi$

\subsubsection{Direct Numerical Method for computing $A_{d B}$}

In the previous section, we derived an approximated expression $\lambda_{\text {est }}$ for the optimal design parameter $\lambda_{\text {opt }}$. Another way of finding the optimal parameter is simply to find the coordinates of the saddle point of the surface response of the FRF (see fig. 2). Those coordinates can be obtained by solving the following two equations for $\omega$ and $\lambda$ :

$$
\frac{\partial H}{\partial \omega}=0, \quad \frac{\partial H}{\partial \lambda}=0 .
$$

Computing the derivatives in equations (17) leads to the following equations:

$$
\begin{aligned}
2 \omega^{6}+A \omega^{4}+B \omega^{2}+C & =0, \\
D \lambda^{2}+E \lambda-F & =0,
\end{aligned}
$$

with:

$$
\begin{array}{ll}
A=\left((1+\mu)^{2}+3\right) \lambda^{2}+4 \xi \mu \lambda+2\left(2 \xi^{2}-1\right) & , D=2 \xi \\
B=2(1+\mu)^{2} \lambda^{4}+8 \xi \mu \lambda^{3}+4\left(2 \xi^{2}-1\right) \lambda^{2} & , E=-2(2+\mu) \omega^{2}+2, \\
C=2\left(2 \xi^{2}-(1+\mu)\right) \lambda^{4} & , F=2 \xi \omega^{2}
\end{array}
$$

The system of Eqs.(18-19) can be solved numerically using classical root finding algorithm (e.g. Newton Raphson algorithm) to yield the optimal values of the damping coefficient $\lambda_{\text {opt }}$. Figure 4 depicts $\lambda_{\text {opt }}$ and $\lambda_{\text {est }}$ as function of the mass ratio for different values of $\xi$. We recall that the value of $\lambda_{\text {est }}$ is independent of the parameter $\xi$, therefore there is only one curve for different values of the primary damping ratio $\xi$ ). Two important conclusions can be drawn from figure 4. First, one can see that the estimation method for the design parameter $\lambda_{\text {est }}$, presented in the previous section, is very reliable, especially for low primary damping $(\xi$ 

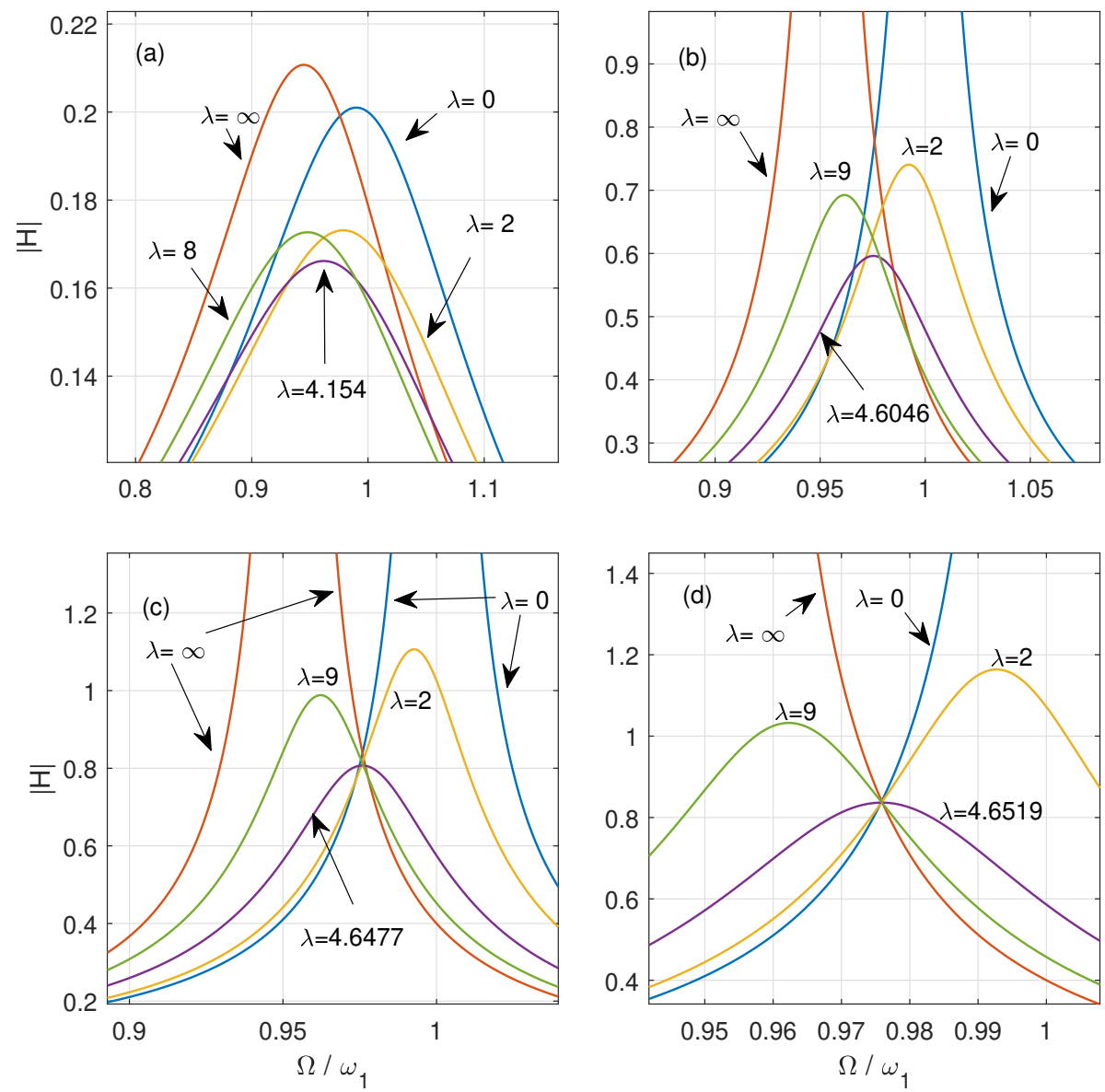

Figure 5: Frequency response function of the primary mass for different values of $\xi$ and $\lambda$. (a): $\xi=0.1,(\mathrm{~b}): \xi=0.01,(\mathrm{c}): \xi=10^{-3}$ and $(\mathrm{d}) \xi=10^{-4}$.

small). Second, using a first order Taylor expension of Eq.(12), one notes that the evolution of the optimal parameter is close to linear with relation to the mass ratio $\mu$ :

$$
\lambda_{\text {est }} \approx 1-\frac{3}{4} \mu
$$

This behaviour indicates the high robustness of $\lambda_{\text {opt }}$ and $\lambda_{\text {est }}$ when $\mu$ is varied.

Figure 5 depicts the modulus of the $\mathrm{FRF}|H(\omega)|$ for several values of $\lambda$ and for different damping values of the primary system $\xi$. One sees that the solution of Eq.(18) actually provides an optimal value for parameter $\lambda_{\text {opt }}$, since the associated FRF (violet curves) have the lowest maximum amplitude.

Figure 6 depicts the comparison between the exact value of $A_{d B}$, computed by the numerical method, and its estimated value obtained in the previous section. It can be seen that the analytical estimation of $A_{d B}$ in Eq. (12) is accurate for all values of $\xi$ up to at least $\xi=0.1$.

One can notice that the efficiency $A_{d B}$ of the linear Lanchester damper, if it has been carefully optimized with Eqs. (12) or (20), depends on only one parameter: the mass ratio $\mu$. 


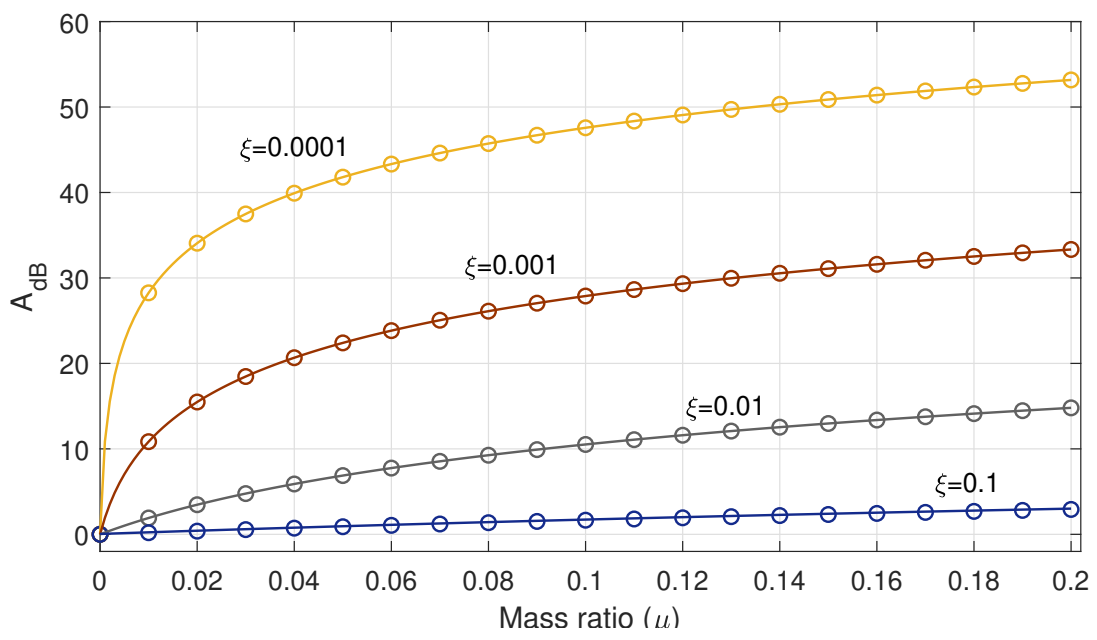

Figure 6: Comparison between numerical and analytical values of $A_{d B}$ as a function of mass ratio $(\mu)$ for several values of $\xi$. Continuous lines $(-)$ relate to the numerical method and circles (o) relate to the analytical method
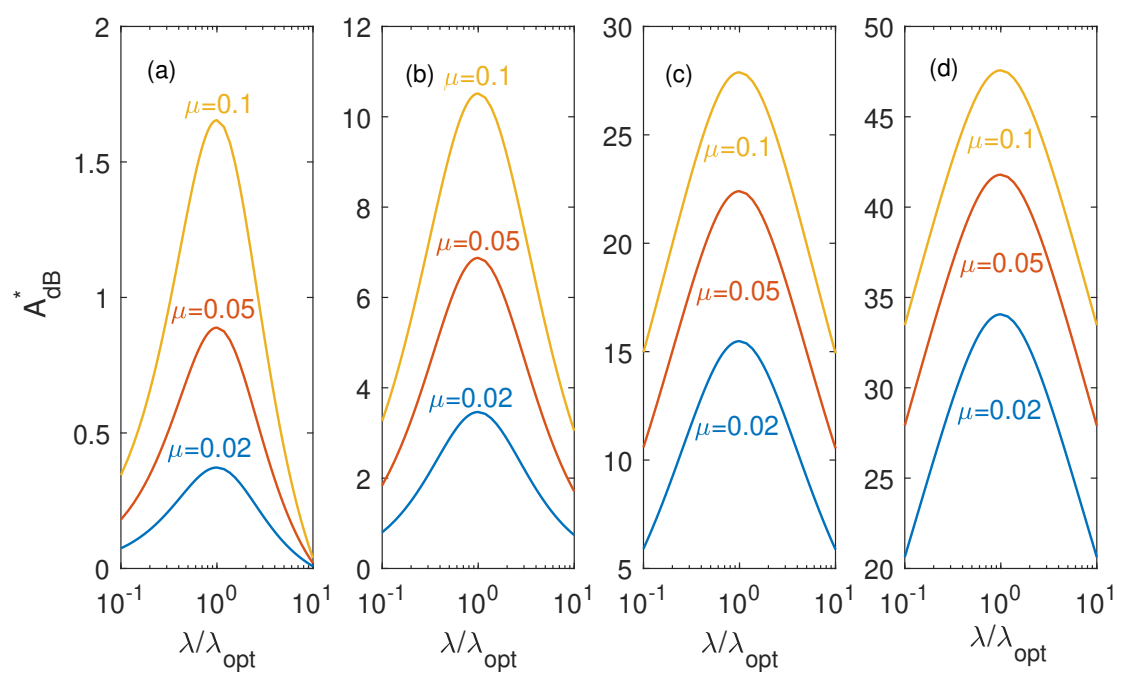

Figure 7: $A_{d B}^{*}$ as function of deviation of the design parameter from its optimal value $\left(\lambda / \lambda_{\text {opt }}\right)$ for several values of $\xi$ and $\mu$. (a): $\xi=0.1,(\mathrm{~b}): \xi=0.01$, (c): $\xi=10^{-3}$ and (d) $\xi=10^{-4}$.

\subsection{Robustness of the Linear Damper}

In order to study the robustness of the absorber, we define another property, denoted $A_{d B}^{*}$, which is essentially the same as $A_{d B}$ (see Eq.(8)) except that it is not computed necessarily with the optimal value of $\lambda$. Figure 7 shows the value of $A_{d B}^{*}$ for a reasonable interval of $\lambda$ around the optimum point $\lambda_{\text {opt }}$. For a given mass ratio, the changes in $A_{d B}^{*}$ is bigger for systems having low primary damping ratio. Also, for fixed primary damping, system with lower mass ratios have smaller changes in $A_{d B}$.

Properties of the viscous element in the system maybe subjected to change during its life time. However, referring to figure 8 , one see that a $20 \%$ variation of the $\lambda$ parameter around its optimal values results in an efficiency decrease of about $2 \%$ in the worst case (for $\xi=10^{-4}$ ). 

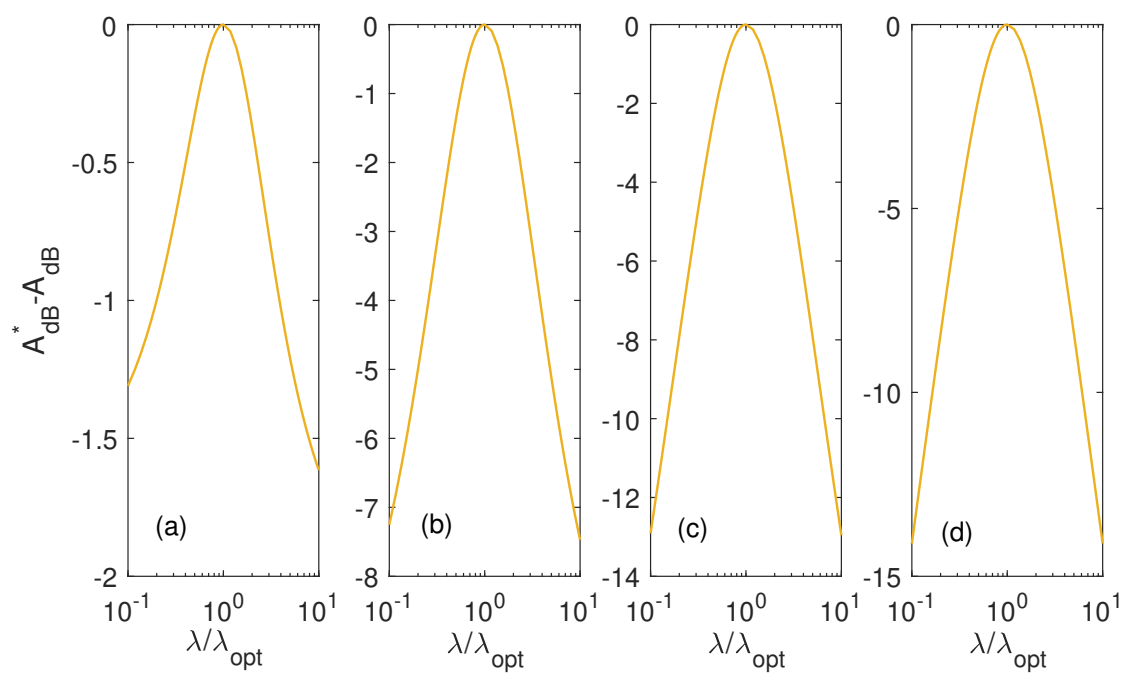

Figure 8: $A_{d B}^{*}-A_{d B}$ as function of deviation of $\lambda_{\text {opt }}$ for several values of $\xi$ and $\mu$. (a): $\xi=0.1$, (b): $\xi=0.01,(\mathrm{c}): \xi=10^{-3}$ and (d) $\xi=10^{-4}$.

In other cases $\left(\xi=10^{-3}, 10^{-2}, 10^{-1}\right)$ the efficiency drop is less than $0.85 \%$. Therefore one can consider that small changes in $\lambda$ will not cause any dramatic decrease in the performance of damper, which can therefore be considered as very robust.

\section{Non Linear Lanchester Damper}

In this section, we consider a non linear Lanchester absorber which is composed of an additional mass connected to the main structure with dry friction force. As before, the primary system is a mass-spring-viscous damper system which is connected to the smaller mass which now slides on the surface of the primary mass (see figure 9). Due to relative displacement, friction force will dissipate energy from the system and reduce the resonance amplitude. In the following sections we will investigate the efficiency of such non linear vibration dampers.

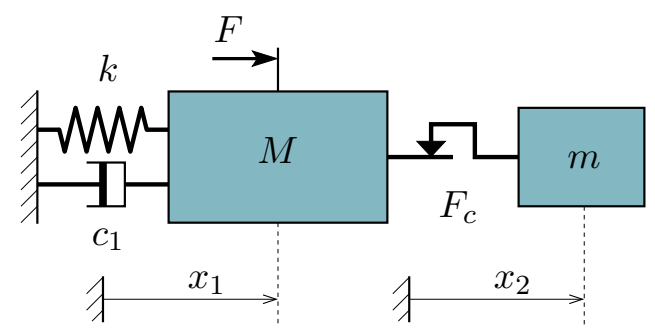

Figure 9: Schematics of a non linear Lanchester absorber attached to a primary system of mass $M$ 


\subsection{Equations of motion}

The equation of motion of the system depicted in figure 9 can be obtain by applying newton's law, which results in the following:

$$
\begin{aligned}
M \ddot{x}_{1}+c_{1} \dot{x_{1}}+k x_{1}+f_{c} & =F \sin (\Omega t), \\
m \ddot{x_{2}}-f_{c} & =0,
\end{aligned}
$$

where $f_{c}$ is the Coulomb's dry friction force between the two masses, defined as function of the relative velocity $\left(\dot{x}_{d}=\dot{x}_{2}-\dot{x}_{1}\right)$ :

$$
\left\{\begin{array}{rrrrr}
f_{c} & & -F_{c} & & \dot{x_{d}}>0 \\
f_{c} & = & F_{c} & & \dot{x_{d}}<0 \\
f_{c} & \in & {\left[-F_{c}, F_{c}\right]} & & \dot{x_{d}}=0
\end{array}\right.
$$

The previous non linear force can be rewritten shortly using the sign function as $f_{c}=$ $F_{c} \operatorname{sign}\left(\dot{x}_{d}\right)$. Using the same procedure as in section 2, one obtains the equation of motion under non dimensional form as :

$$
\left\{\begin{aligned}
\ddot{\bar{x}}_{1}+\mu \ddot{\bar{x}}_{2}+2 \xi \dot{\bar{x}}_{1}+\bar{x}_{1} & =\sin (\omega \bar{t}) \\
\mu \ddot{\bar{x}}_{2}+\lambda \operatorname{sign}\left(\dot{\bar{x}}_{d}\right) & =0
\end{aligned}\right.
$$

with:

$$
\omega=\frac{\Omega}{\omega_{1}}, \quad \lambda=\frac{F_{c}}{F}, \quad a=\frac{F}{k}, \quad \overline{x_{1}}=\frac{x_{1}}{a}, \quad \overline{x_{2}}=\frac{x_{2}}{a}, \quad \bar{t}=\omega_{1} t .
$$

Note that the parameter $\lambda$ is the non linear counterpart of the parameter $\lambda$ defined for the linear damper, with the difference that it now depends on the amplitude of excitation force. For brevity sake, we will keep using the notation $\lambda$ for the non linear parameter as well.

\subsection{Direct Steady-state solution}

In this section, we look for the steady state solutions of Eq.(23) in order to study the frequency response behaviour of the non linear system. Depending on the amplitude and frequency of the exciting force, three types of solutions are to be expected: (i) slip-only, (ii) stick- only and (iii) stick-slip responses. Since the non linear force is an odd function of velocity, the response is expected to be odd as well (a proof is presented in A). Thus, only half a period will be considered for the study of the steady states, as proposed in [20]. After describing the three types of motion, we will look for transition frequencies from one type of motion to the other. This will allow to compute the frequency response and consequently the optimal design parameter for the non linear absorber.

\subsubsection{Stick-only response}

Here the damper is fully stuck to the primary structure $\left(x_{2}=x_{1}, x_{d}=0\right)$ and the problem is linear and consists of a single dof system with a mass of $1+\mu$. Equation of motion in this response type is given by the following:

$$
(1+\mu) \ddot{\bar{x}}_{1}+2 \xi \dot{\bar{x}}_{1}+\bar{x}_{1}=\sin (\omega \bar{t}) .
$$


The response of the system is then a pure sine function for $x_{1}(t)=x_{2}(t)$ with amplitude:

$$
\hat{A}=\frac{1}{1+\mu} \frac{1}{\sqrt{\left(\hat{\omega}_{1}^{2}-\omega^{2}\right)^{2}+4 \hat{\xi}^{2} \hat{\omega}_{1}^{2} \omega^{2}}} .
$$

where

$$
\hat{\omega}_{1}=\sqrt{\frac{1}{1+\mu}}, \quad \hat{\xi}=\frac{\xi}{\sqrt{1+\mu}} .
$$

$\hat{\omega}_{1}$ and $\hat{\xi}$ are respectively the dimensionless natural frequency and the damping ratio of the system with the two masses fully stuck.

\subsubsection{Stick-slip response}

Depending on the parameters of the system, mainly if the maximum friction force $\left(F_{c}\right)$ is in the same order as the excitation force $(F)$ and if the excitation frequency is far from the natural frequency of the primary system, the two masses will both lock and unlock during half a period leading to a stick-slip response. Here only one stick phase per half period will be considered. Figure 10 shows the general form of different variables in this response type over one period of excitation.

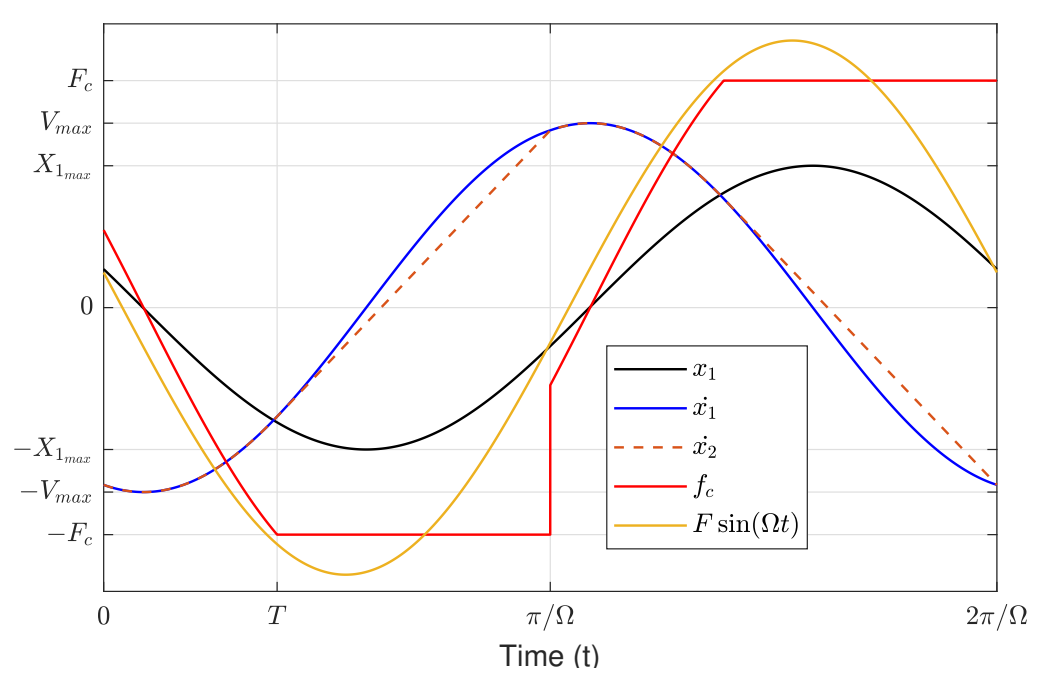

Figure 10: Representation and characteristics of a stick-slip response for the two dof system of fig.9. Arbitrary units for $y$-axis.

The beginning of the period is chosen to be the time at which the two masses start to lock $\left(x_{2}=x_{1}, x_{d}=0\right)$. Translating time origin to this instant can be done by adding a (yet unknown) phase $\phi$ in the forcing term of Eq. (23) which now becomes $\sin (\omega \bar{t}+\phi)$. Locking is possible only when the velocity of both masses are equal. The masses will unlock when the force required to keep them connected reaches the maximum value of friction force $\left(F_{c}\right)$.

Consider $T$ as the unlocking time; then, the motion over one half period consists of two parts: (i) for $0<t<T$ the masses are connected and (ii) for $T<t<\frac{\pi}{\Omega}$ the masses move 
separately. In the first part, the system could be considered as a linear single degree of freedom system excited with a force $(F \sin (\Omega t+\phi))$. In the second part, the motion of $M$ is a result of both the excitation force and the friction force, whereas the dynamic of mass $m$ is only imposed by the friction force. In the following we will derive the expression for each part of the motion.

Part 1 (Stick): As mentioned before, in this part both masses are locked together and can thus be considered as one $\left(x_{1}=x_{2}\right)$. The dimensionless equation of motion for this part is given as:

$$
(1+\mu) \ddot{\bar{x}}_{1}+2 \xi \dot{\bar{x}}_{1}+\bar{x}_{1}=\sin (\omega \bar{t}+\phi) .
$$

The solution of the previous equation is the sum of a transient response and a steady states response:

$$
\bar{x}_{1}(\bar{t})=\hat{A} \sin (\omega \bar{t}+\hat{\alpha}+\phi)+e^{-\hat{\xi} \hat{\omega}_{1} \bar{t}}\left[b \sin \left(\hat{\omega}_{d} \bar{t}\right)+a \cos \left(\hat{\omega}_{d} \bar{t}\right)\right], \quad(0 \leqslant \bar{t} \leqslant \bar{T})
$$

where $\hat{A}$ is defined by Eq. (25) and

$$
\hat{\omega}_{d}=\hat{\omega}_{1} \sqrt{1-\hat{\xi}^{2}}, \quad \hat{\alpha}=-\operatorname{atan} \frac{2 \hat{\xi} \hat{\omega}_{1} \omega}{\hat{\omega}_{1}^{2}-\omega^{2}} .
$$

The constant $a$ and $b$ are obtained by taking into account the initial conditions:

$$
\begin{aligned}
a & =X_{0}-\hat{A} \sin (\hat{\alpha}+\phi), \\
b & =\frac{1}{\hat{\omega}_{d}}\left[V_{0}+\hat{\xi} \hat{\omega}_{1}\left(X_{0}-\hat{A} \sin (\hat{\alpha}+\phi)\right)-\hat{A} \cos (\hat{\alpha}+\phi)\right],
\end{aligned}
$$

with $X_{0}$ and $V_{0}$ being $\bar{x}_{1}(0)$ and $\dot{\bar{x}}_{1}(0)$ respectively.

Part 2 (Slip): In this part, the two masses move separately. The initial conditions for this part of the motion are the final values of the displacement and the velocity of the stick part (part 1). Let's denote the initial displacement as $X_{1}=\bar{x}_{1}(\bar{T})$ and the initial velocity as $V_{1}=\dot{\bar{x}}_{1}(\bar{T})$.

In the following we find the motion of each mass. We first consider the motion of the secondary mass $m$ subjected to the following equation of motion:

$$
\mu \ddot{\bar{x}}_{2}=\lambda
$$

which allows to compute the velocity of the secondary mass directly as (for $\left.\bar{T} \leq \bar{t} \leq \frac{\pi}{\omega}\right)$ :

$$
\dot{\overline{x_{2}}}(\bar{t})=\frac{\lambda}{\mu}(\bar{t}-\bar{T})+V_{1} .
$$

We now consider the motion of the primary mass $M$ subjected to the following equation of motion:

$$
\ddot{\overline{x_{1}}}+2 \xi \dot{\overline{x_{1}}}+\overline{x_{1}}+\lambda=\sin (\omega \bar{t}+\phi) .
$$

A solution of equation (32) can be expressed as follows for $\bar{T} \leqslant \bar{t} \leqslant \frac{\pi}{\omega}$ :

$$
\overline{x_{1}}(\bar{t})=A \sin (\omega \bar{t}+\alpha+\phi)-\lambda+e^{-\xi(\bar{t}-\bar{T})}\left[d \sin \left(\omega_{d}(\bar{t}-\bar{T})\right)+c \cos \left(\omega_{d}(\bar{t}-\bar{T})\right)\right],
$$

where: 


$$
A=\frac{1}{\sqrt{\left(1-\omega^{2}\right)^{2}+4 \xi^{2} \omega^{2}}}, \quad \alpha=-\tan ^{-1}\left(\frac{2 \xi \omega}{1-\omega^{2}}\right), \quad \omega_{d}=\sqrt{1-\xi^{2}} .
$$

The constants $c$ and $d$ can be obtained using the final condition of the stick phase:

$$
\begin{aligned}
& c=X_{1}-A \sin (\omega \bar{T}+\alpha+\phi)+\lambda \\
& d=\frac{1}{\omega_{d}}\left[V_{1}+\xi\left(X_{1}-A \sin (\omega \bar{T}+\phi+\alpha)+\lambda\right)-A \cos (\omega \bar{T}+\phi+\alpha)\right] .
\end{aligned}
$$

As a result, one sees that there are 4 unknown parameters in the description of the stick-slip motion: $\left(T, \phi, X_{0}, V_{0}\right)$. These four parameters can be obtained by solving the following set of equations:

$$
\begin{aligned}
\mu \ddot{\bar{x}}_{1}(\bar{T}) & =\lambda, \\
\dot{\bar{x}}_{1}\left(\frac{\pi}{\omega}\right) & =-V_{0}, \\
\dot{\bar{x}}_{2}\left(\frac{\pi}{\omega}\right) & =-V_{0}, \\
\bar{x}_{1}\left(\frac{\pi}{\omega}\right) & =-X_{0} .
\end{aligned}
$$

The above equations are related to the following conditions: (i) the connecting force between two masses should be equal to the maximum friction force at $\bar{t}=\bar{T}$ (Eq. (35a)), (ii) and (iii) the velocity of both masses should be equal and equal to $-V_{0}$ at $\bar{t}=\frac{\pi}{\omega}$ (Eq. (35b) and (35c)),

(iv) the displacement of mass $M$ should be equal to $-X_{0}$ at $\bar{t}=\frac{\pi}{\omega}(\mathrm{Eq}$. (35d)). Solving this set of equations allows to finds the parameters $\left(T, \phi, X_{0}, V_{0}\right)$ and to reconstruct a stick-slip motion of over a period. This set of non linear equations is solved using iterative algorithms provided by fsolve function in Matlab software.

\subsection{3 slip-only response}

Here we suppose that the two masses will not lock to each other during the period, as depicted on figure 11.

The expression for the slip-only solution can be obtained as a particular case of stick-slip response studied previously. Indeed, the slip motion is similar to the second part of the stick-slip response in Eq.(33). The only difference would be to set the value of $T$ to zero $(T=0)$.

The equations to be solved to find the three unknown parameters $\left(\phi, X_{0}\right.$ and $\left.V_{0}\right)$ in this response type are of the same type as in the stick-slip situation except that one have to be removed. The equation to be kept are Eqs.(35b) , (35c) and (35d).

As the main objective is to observe the amplitude of the frequency response over a frequency interval around the resonance, the computation are carried out sequentially in increasing order of frequency with a fixed frequency step. At each point, the set of solutions is solved and the results are then used as an initial guess for the next step. For the initial point of the frequency response, it can be obtained using a linear computation (for the fully stick case, away from the resonance) or it can be found using a time integration algorithm (e.g. Runge Kutta). 


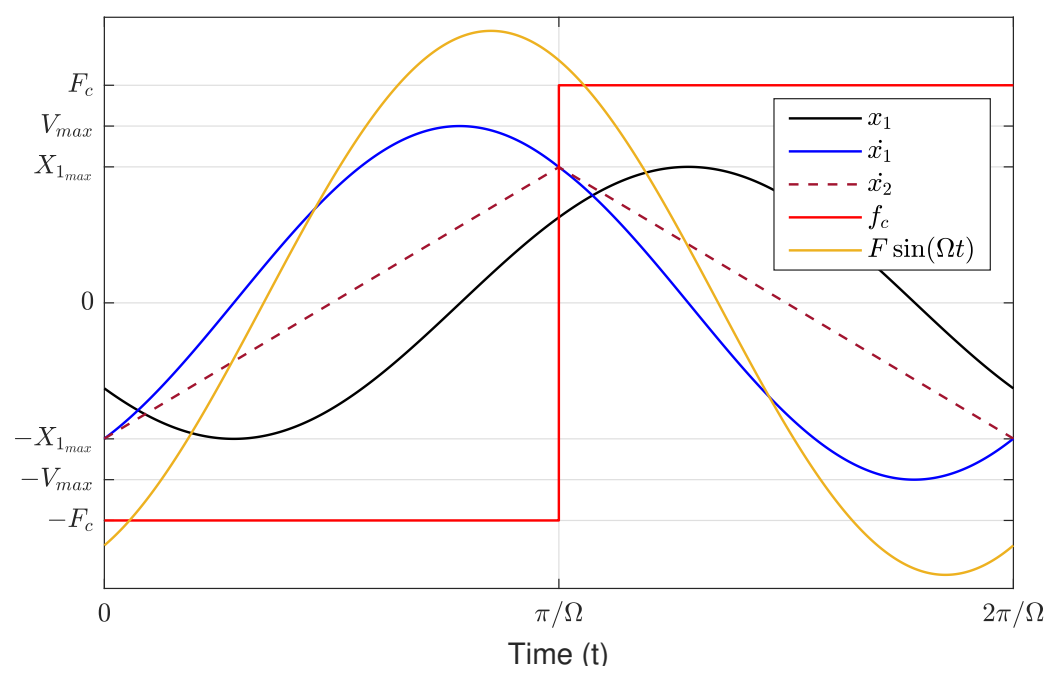

Figure 11: Representation and characteristics of a slip only response for the two dof system of fig.9. Arbitrary units for $y$-axis.

\subsubsection{Switching points}

Now that the different types of steady state motion have been described, it is important to find out how the change occurs between one type to another when the excitation frequency changes (see e.g. fig.12).

For very low frequencies, the two masses are connected to each other $\left(x_{1}=x_{2}\right)$, acting as a single mass up to a point where the required connecting force between them is equal to the maximum available friction force. Following Eqs (21b) and (23), it can be written:

$$
m \ddot{x}_{2}=F_{c} \quad \Rightarrow \quad \mu \ddot{\bar{x}}_{2}=\lambda .
$$

Since the system acts as a one degree of freedom linear system solution of Eq. (24), $\bar{x}_{2}=\bar{x}_{1}$ is a pure sine function with amplitude $\hat{A}$ defined by Eq. (25). Consequently, at the switching point, one has:

$$
\mu \omega^{2} \hat{A}=\lambda
$$

The above equation is a non linear equation w.r.t the excitation frequency $\omega$. The first positive root of this equation corresponds to the transition point between stick and stick-slip motion, and it can be obtained using numerical root finding algorithm.

When the excitation frequency increases, another transition point can appear corresponding to the transition between stick-slip and fully slipping motion. This transition happens when the solution of Eqs.(35a) to (35d) yield negative value for the variable $\bar{T}$. Note that this transition may not exists, particularly for systems with high value of $\lambda$.

When the excitation frequency increases even more, the transitions happen in reversed order (i.e. from fully slipping to stick-slip motion and from stick-slip to fully stick motion). The transition from fully slipping to stick-slip happens when the solution of Eqs.(35a) to (35d) corresponds to a velocity which is not always negative in the first half-period, and thus out of the hypothesis of the previous section (According to Eqs. (35b) and (35c), the final value of the velocity in both masses must be the opposite value of their initial value in a half-period). 
From this frequency on, the motion is under stick-slip form. This stick-slip motion will be valid until the excitation frequency reaches the maximum positive root of Eq.(37). After this point, the two masses get fully stuck again.

\subsubsection{Frequency Response plot}

Figure 12 shows an example of frequency response of the primary mass illustrating the position of the transition points between the different types of motion for a specified system over a wide range of excitation frequency. For each frequency $\omega$, the steady state response function is computed and the maximum displacement of the primary mass is extracted from the solution and termed hereafter as $X_{1 M}$.

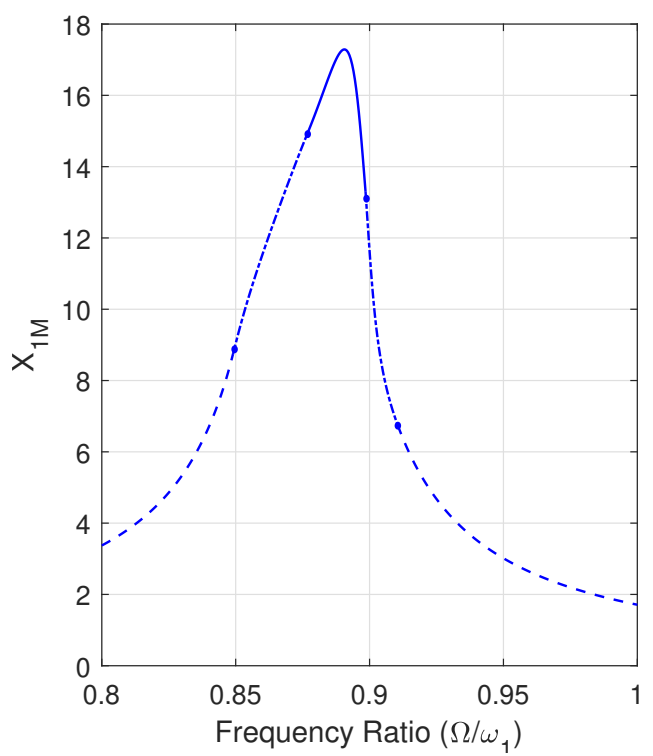

Figure 12: Frequency response for the primary system of figure 9 with $\xi=0.01, \mu=0.1$ and $\lambda=0.7$. Continuous line shows slip only response, dash-dotted lines shows stick-slip response and dashed lines shows the stick-only response type. circles (o) indicate switching points between response types.

\subsubsection{Results}

Steady state solutions have been computed for several values of $\xi$ and $\lambda$ over a wide range of excitation frequency for systems having parameter $\mu$ set to 0.05. Dimensionless frequency response plots of displacement of the primary mass $(M)$ are shown in figures 13 and 14 . Those figures show several aspects of a non linear Lanchester damper.

First, as expected, it can be seen that by increasing the friction force in the system (increasing $\lambda$ ), the response of the system gets closer and closer to the response of a system where the two masses are connected $(\lambda=\infty)$. In this case, the system has only one degree of freedom and shows a classical linear resonant response of resonance frequency close to $\hat{\omega}_{1}$ 

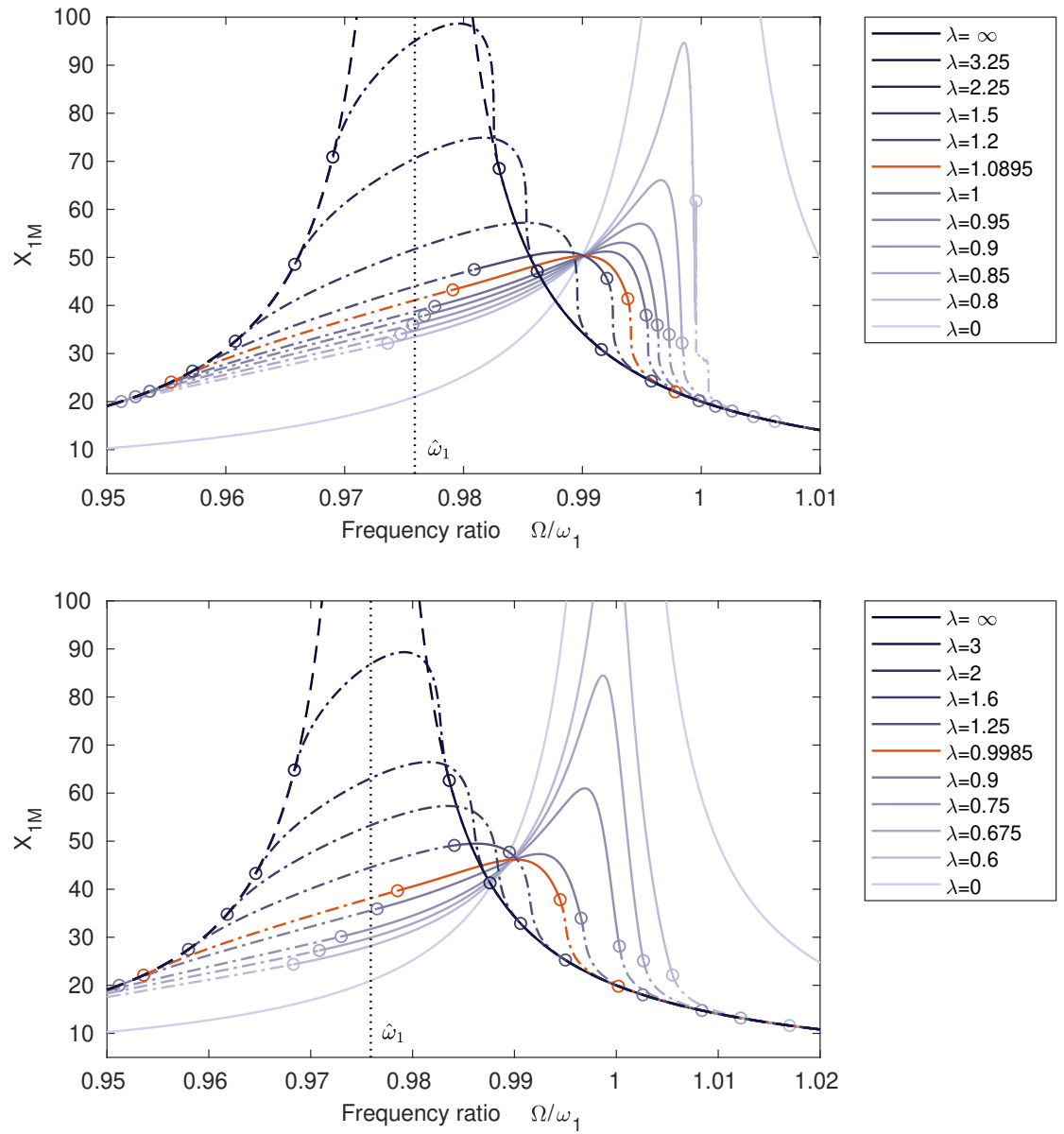

Figure 13: Frequency response of the primary mass for $\xi=10^{-4}$ (top plot) and $\xi=10^{-3}$ (bottom plot) with $\mu=0.05$. Continuous line shows slip-only response, dash-dotted lines shows stick-slip response and dashed lines shows the stick-only response type. Circles (o) indicate switching points between response types. The colors are related to a given value of $\lambda$, shown in the legend. 

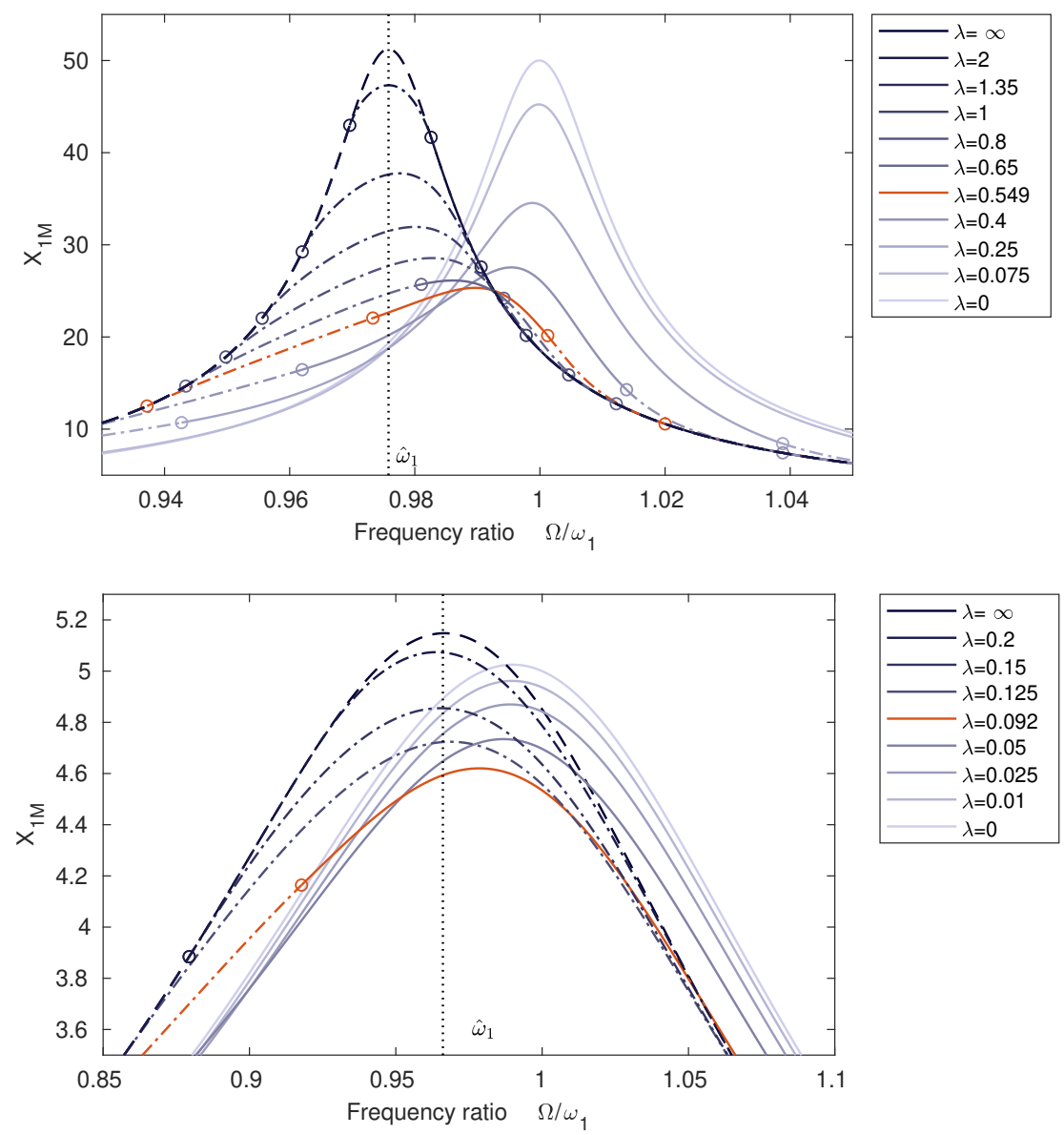

Figure 14: Frequency response of the primary mass for $\xi=0.01$ (top plot) and $\xi=10^{-1}$ (bottom plot) with $\mu=0.05$. Continuous line shows slip-only response, dash-dotted lines shows stick-slip response and dashed lines shows the stick-only response type. circles (o) indicate switching points between response types. The colors are related to a given value of $\lambda$, shown in the legend. 
(Eq. (26)), depicted on Figs. 13 and 14 with dashed dark blue lines. Also, when the friction force increases, the frequency interval corresponding to a fully slipping response shortens.

Second, one sees that when the parameter $\lambda$ increases from low to high values, the maximum amplitude of the response (of the primary mass) decreases to a minimum, and then increases again. This indicates that in non linear Lanchester damper, as well as for a linear Lanchester damper, there exists an optimal value for $\lambda$ which minimizes the maximum amplitude of the primary mass. For each given system presented in Figures 13 and 14, the frequency response corresponding to the optimal value of $\lambda\left(\lambda_{\text {opt }}\right)$ is shown in orange. The next step is to compute the efficiency of such a non linear Lanchester damper and to compare it with the one of a linear Lanchester damper.

\subsection{Efficiency of non linear damper}

In this section we focus on the efficiency of a non linear Lanchester damper. The simulations presented in this section have been carried out for several values of the mass ratio $\mu$ going from $\mu=0.0025$ to $\mu=0.2$. For each value of $\mu$ the solution has been computed over a wide range of excitation frequency for several value of $\lambda$ in order to find the optimal value of this design parameter $\left(\lambda_{\mathrm{opt}}\right)$.

The efficiency of the non linear damper is characterized by the quantity $A_{d B}$ which has the same definition as in the linear case (see Eq.(8)). The efficiency of the non linear Lanchester damper is depicted on figure 15 , for several values of the mass ratio $\mu$ and several value of the primary damping $\xi$. One can see that for a given mass, the efficiency of the non linear damper is much higher in systems having low primary damping ratio ( i.e small $\xi$ ).

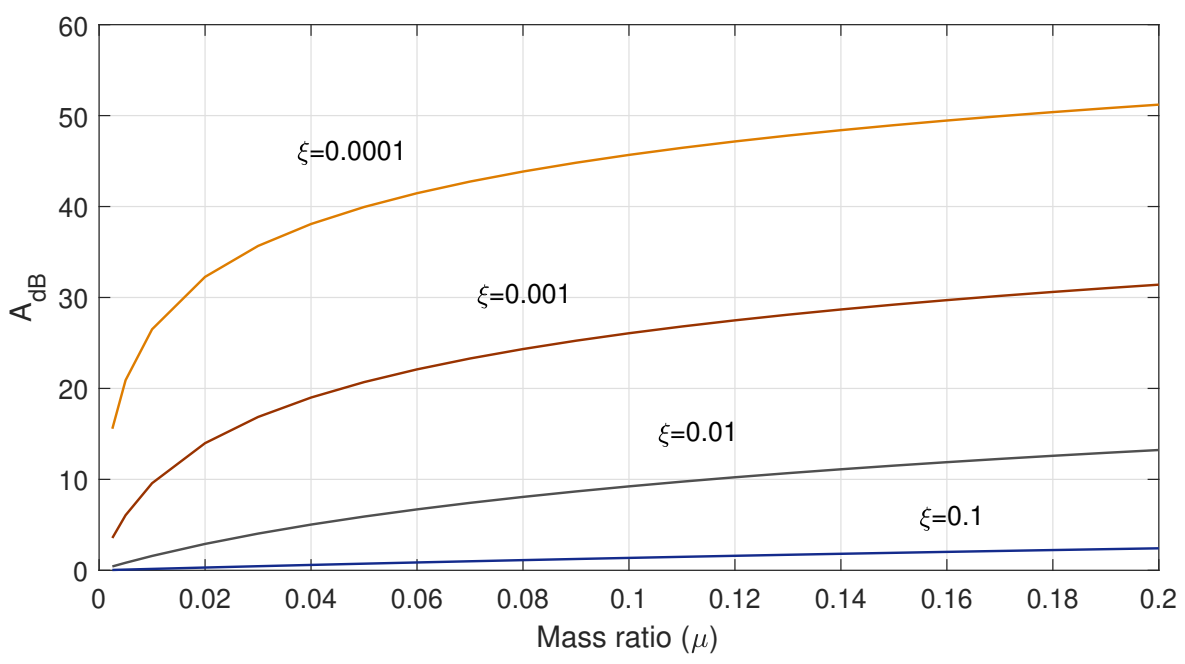

Figure 15: Efficiency $A_{d B}$ of the non linear Lanchester damper, as a function of the mass ratio $\mu$ for several values of $\xi$.

The optimal value of $\lambda_{\text {opt }}$ as a function of the mass ratio and primary damping ratio is depicted on figure 16. One can conclude from Figure 16 that for a given mass ratio, the required friction force to meet the optimal value of $\lambda$ is greater for system having low primary damping ratio. Figure 16 also shows that, for low primary damping ratio, the optimal value of $\lambda$ is almost constant with relation to the mass ration $\mu$. 


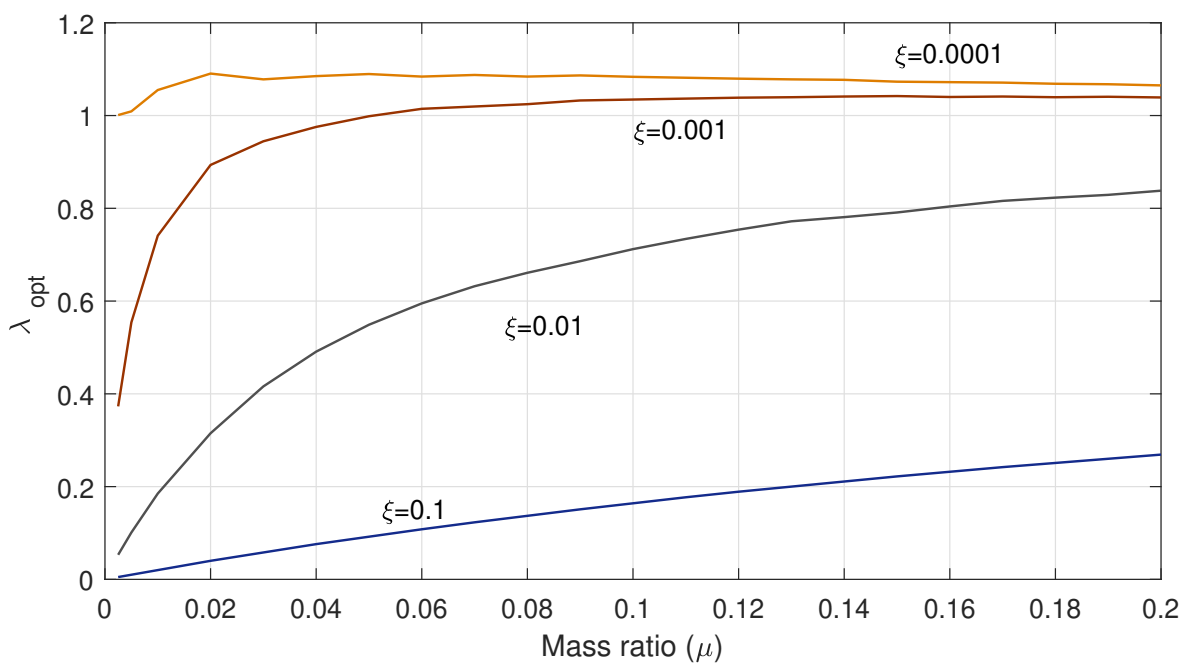

Figure 16: Optimal values of $\lambda$ for the non linear Lanchester damper, as a function of the mass ratio $\mu$, for several values of $\xi$.

\subsection{Robustness of non linear damper}

In this section we investigate the robustness of the non linear Lanchester damper. The procedure is the same as in the linear case already presented in the first section of this paper. Recall that the robustness is evaluated using the quantity $A_{d B}^{*}$ which is essentially the same as $A_{d B}$ except that it is not necessarily computed with the optimal design value $\left(\lambda_{\text {opt }}\right)$.

In order to evaluate the robustness of the non linear Lanchester damper, the mass ratio has been fixed to $\mu=0.1$, an the quantity $A_{d B}^{*}$ has been computed for several values of $\lambda$ around the optimal point $\lambda_{\text {opt }}$. The results are shown on figure 17 .

It can be seen that the efficiency drops when $\lambda$ is varied. It is noteworthy that systems with higher primary damping experience a higher drop in performance. However, the system can be seen as quite robust since a $20 \%$ variation in $\lambda$ around $\lambda_{\text {opt }}$ induces a drop in efficiency of at most $6.5 \%$. System having lower primary damping ratio (lower $\xi$ ) seems to be more robust, especially when $\lambda$ is greater than $\lambda_{\text {opt }}$ (in those cases, the efficiency decreases slower for $\lambda>\lambda_{\text {opt }}$ compared to the decrease for $\lambda<\lambda_{\text {opt }}$ ). 

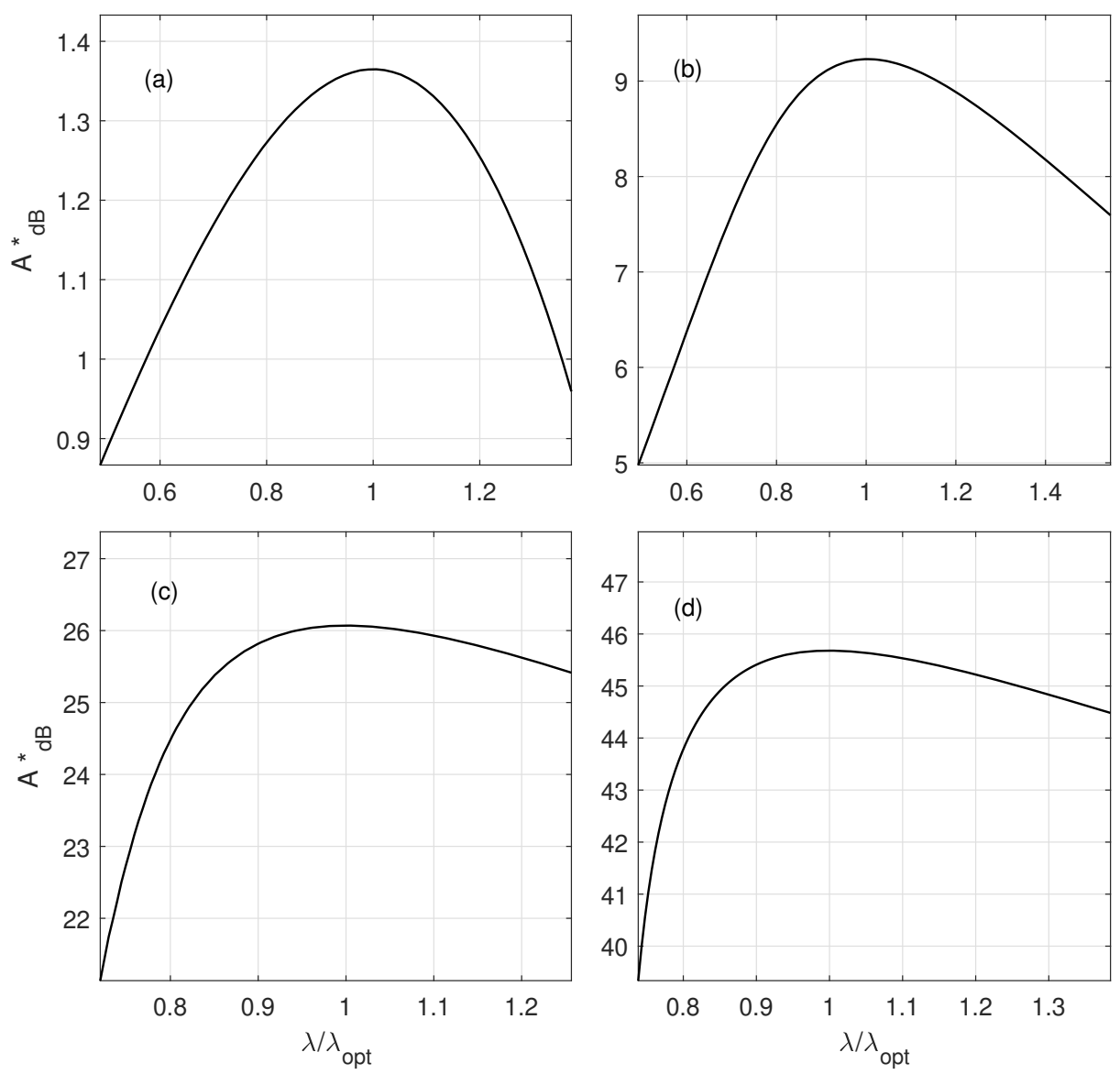

Figure 17: Efficiency $\left(A_{d B}^{*}\right)$ as function of deviation of $\lambda$ from its optimal value $\left(\lambda_{\text {opt }}\right)$ for different values of $\xi$, with $\mu=0.1$. (a): $\xi=10^{-1}$, (b): $\xi=10^{-2}$, (c): $\xi=10^{-3}$, (d): $\xi=10^{-4}$

\section{Comparison and discussion}

In this section we compare and discuss the results obtained for a linear and non linear Lanchester damper. The comparison is done with relation to the efficiency factor $A_{d B}$, and is depicted on Figure 18, where we plotted the efficiency of the linear damper along with the efficiency of the non linear damper. Overall, for a given primary system, it is evident that the efficiency of the linear damper is slightly better that the efficiency of the non linear damper. The discrepancy between the efficiency is bigger for smaller values of the primary damping. When the primary damping increases, the efficiency of the two dampers (linear and non linear) are globally equivalent.

Another point of comparison can be drawn by comparing the robustness of the two kinds of damper. Figure 19 depicts the evolution of the efficiency as a function of the deviation of design parameter $\lambda$ from its optimal value $\lambda_{\text {opt }}$ for linear and non linear dampers, and for several values of the primary damping $\xi$. In this figure, it can be seen clearly that the linear damper is much more robust compared to the non linear damper.

Following this comparison, we can conclude that the linear Lanchester damper outperforms the non linear Lanchester damper since it always has a better efficiency, and is much more robust. Moreover, in the linear case, the optimal parameter $\lambda$ is independent of the excitation 


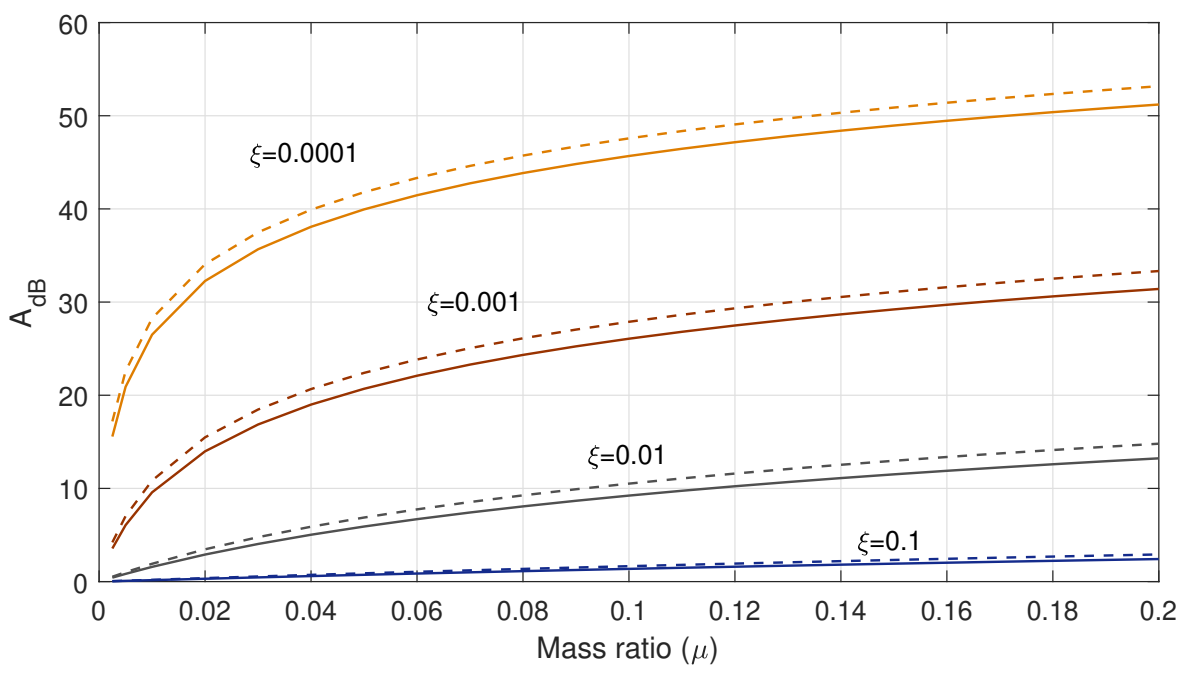

Figure 18: Comparison of the efficiency $A_{d B}$ between linear and non linear dampers, as function of $\mu$, for different values of $\xi$. Continuous lines are related to non linear damper and dashed lines are related to the linear damper.

amplitude, so that it stays optimal regardless to the amplitude of the excitation force. This is another advantage over the non linear damper. Indeed, the optimal $\lambda$ in the non linear case is depending on the force amplitude, and thus has to be changed when the excitation amplitude changes. 

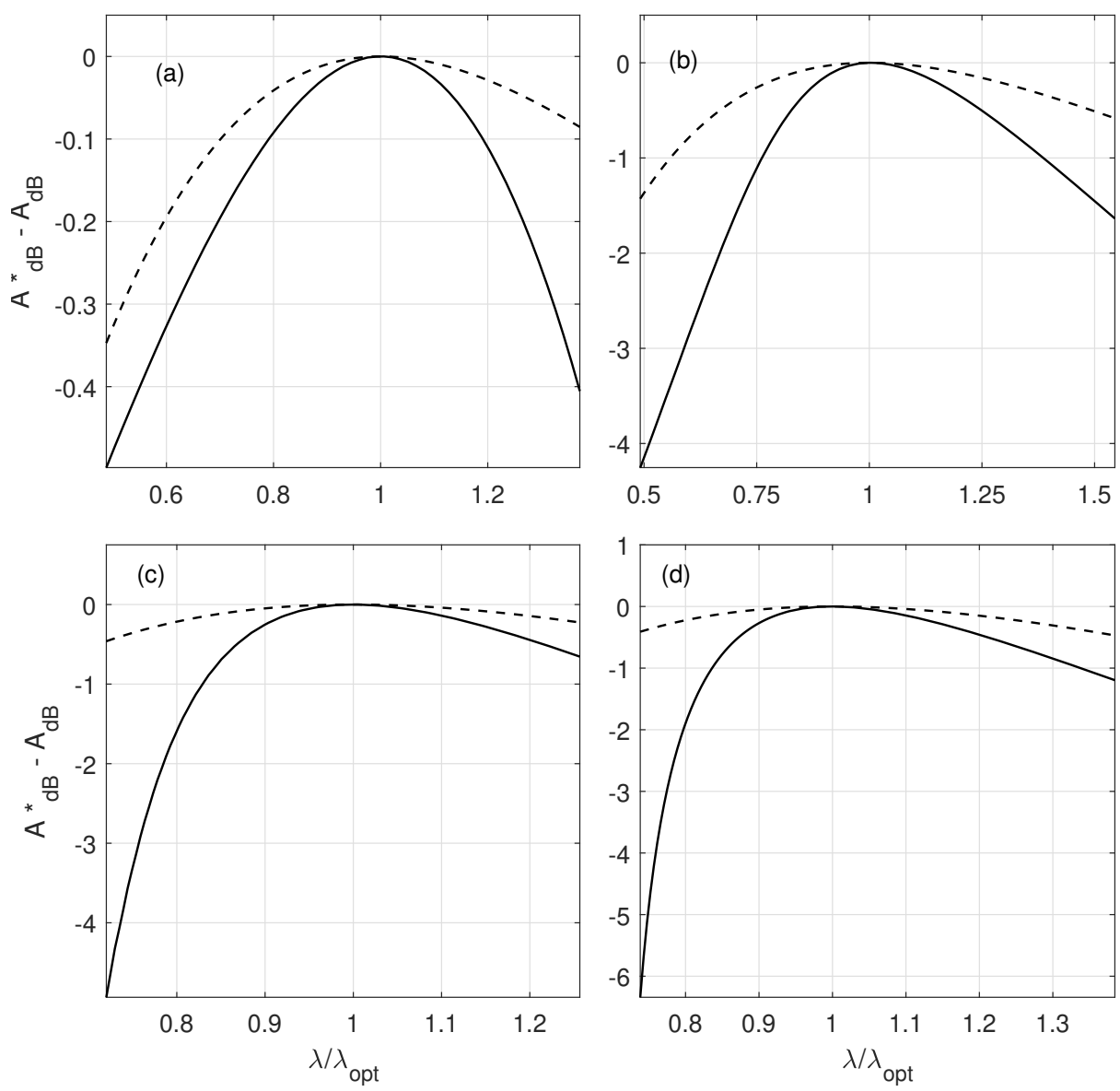

Figure 19: Efficiency drop $\left(A_{d B}^{*}-A_{d B}\right)$ for both linear (dashed lines) and non linear (continuous lines) for different values of $\xi$, with $\mu=0.1$. (a): $\xi=10^{-1},(\mathrm{~b}): \xi=10^{-2}$, (c): $\xi=10^{-3}$, (d): $\xi=10^{-4}$

\section{Conclusion}

In this paper, we studied and compared linear and non linear Lanchester damper in the context of vibration reduction. For both cases methods were developed to tune the damper based on parameters of the primary system. Dimensionless equations are considered in order to generalize the results to many different systems.

For the linear damper, the optimal parameters have been estimated using an analytical approximation for the efficiency. Those estimations have been validated using results coming from a direct numerical method.

For the non linear damper, the optimal parameters have been estimated using a semianalytical procedure. First, the frequency response of the primary system is constructed by considering that three types of solution can be observed: (i) stick-only, (ii) stick-slip and (iii) slip-only. Then the optimal values of the parameters are obtained by searching for the frequency response having the lowest maximum amplitude.

The comparison between linear and non linear dampers is then carried out based on their efficiency and their robustness. Globally, it can be seen that the linear damper outperforms 
the non linear damper both in term of efficiency and robustness. Moreover, the linear damper remains optimal regardless to the amplitude of the excitation force, whereas the optimal parameter for the non linear damper depends on the excitation force level.

Even though linear system performs better, in physical system, dry friction force are almost inevitable, and this study provides reference curves for the study of a primary system coupled to a damper through dry friction force. Those curves can serve as a benchmark for future comparison using fully numerical procedures.

The presented method and the results obtained in this paper can be used as a reference solution for the frequency response of non linear system with dry friction forces. This reference can then be used in order to validate numerical procedures such as the harmonic balance method in the context of dry friction.

\section{A Symmetry in the response curves}

We consider the following harmonically forced dynamical system:

$$
m \ddot{x}+c \dot{x}+k x+f_{\mathrm{nl}}(\dot{x})=f_{\mathrm{e}} \cos \Omega t
$$

where $x(t)$ is a function of time $t$, $\dot{o}$ is the time derivative of $\circ, m, c, k$ are mass, damping and stiffness constants, $f_{\mathrm{nl}}(\dot{x})$ is the non linear internal force and $f_{\mathrm{e}}$ is the external force. We assume that $f_{\mathrm{nl}}(\dot{x})$ is an odd function of $\dot{x}$, which means that for all $\dot{x}$ :

$$
f_{\mathrm{nl}}(-\dot{x})=-f_{\mathrm{nl}}(\dot{x})
$$

As a consequence, its Taylor series expansion around 0 contents only odd terms:

$$
f_{\mathrm{nl}}(\dot{x})=a_{1} \dot{x}+a_{3} \dot{x}^{3}+a_{5} \dot{x}^{5}+\ldots=\sum_{i=1,3,5}^{+\infty} a_{i} \dot{x}^{i},
$$

where the $a_{i}, i \in \mathbb{N}$ are the Taylor coefficients of $f_{\mathrm{nl}}$. If periodic solutions of Eq. (38), in the steady state, are under concern, they can be written as the following Fourier series:

$$
x(t)=x_{0}+\sum_{h=1}^{+\infty}\left(x_{h}^{c} \cos h \Omega t+x_{h}^{s} \sin h \Omega t\right)
$$

where $\Omega=2 \pi / T$ is the frequency of motion, $T$ its period and $\left(x_{h}^{c}, x_{h}^{s}\right)$ are the Fourier coefficients of $x(t)$. In Eq. (38), the harmonics content of $x(t)$ is created by the only non linear term of the equation, the function $f_{\mathrm{nl}}$. Since it is odd, it creates only odd harmonics in $x(t)$. For instance, if $x(t)=\cos (\Omega t+\varphi)=\cos \phi, x^{3}=(3 \cos \phi+\cos 3 \phi) / 4, x^{5}=(10 \cos \phi+5 \cos 3 \phi+\cos 5 \phi) / 16 \ldots$ so that:

$$
x(t)=\cos (\Omega t+\varphi) \quad \Rightarrow \quad f_{\mathrm{nl}}(\dot{x})=\sum_{h=1,3,5}^{+\infty}\left(f_{h}^{c} \cos h \Omega t+f_{h}^{s} \sin h \Omega t\right),
$$

As a consequence, the simplest solution $x(t)$ of Eq. (38) is composed only by odd harmonics:

$$
x(t)=\sum_{h=1,3,5}^{+\infty}\left(x_{h}^{c} \cos h \Omega t+x_{h}^{s} \sin h \Omega t\right),
$$


Even harmonics can still be created after a symmetry breaking bifurcation, a case out of the scope of the present text. Now, we consider the following symmetry property of the $T$-periodic time function $x(t)$ :

$$
\forall t, \quad x(t+T / 2)=-x(t)
$$

so that one half of the period is the inverse mirror of the other half period. Inserting Eq. (44) into Eq. (41), one shows that necessarily all even harmonics of $x(t)$ are zero, so that $x(t)$ has an odd harmonics content (Eq. (43)). The reciprocal rule is also verified: any time periodic function with an odd harmonics content verify the symmetry property (44).

As a conclusion, if $f_{\mathrm{nl}}(\dot{x})$ is an odd function of $\dot{x}$, the harmonics content of the basic (without considering symmetry breaking bifurcations) non linear solution $x(t)$ of Eq. (38) is necessarily odd and the symmetry property (44) is verified.

\section{References}

\section{References}

[1] A. Preumont, Vibration control of active structures, 3rd Edition, Springer, 2011.

[2] J. Ducarne, O. Thomas, J.-F. De, Structural vibration reduction by switch shunting of piezoelectric elements: modelling and optimization, Journal of Intelligent Materials Systems and Structures 21 (8) (2010) 797-816.

[3] O. Thomas, J. Ducarne, J.-F. De, Performance of piezoelectric shunts for vibration reduction, Smart Materials and Structures 21 (1) (2012) 015008.

[4] M. Berardengo, O. Thomas, C. Giraud-Audine, S. Manzoni, Improved resistive shunt by means of negative capacitance: new circuit, performances and multi-mode control, Smart Materials and Structures 25 (7) (2016) 075033.

[5] C. Hammami, E. Balmes, M. Guskov, Numerical design and test on an assembled structure of a bolted joint with viscoelastic damping, Mechanical Systems and Signal Processing 70-71 (2016) 714-724.

[6] B. Morin, A. Legay, J.-F. Deü, Reduced order models for dynamic behavior of elastomer damping devices, Finite Elements in Analysis and Design 143 (2018) 66-75.

[7] S. Krenk, Frequency analysis of the tuned mass damper, Journal of Applied Mechanics 72 (2005) 936-942.

[8] F. W. Lanchester, Damping torsional vibrations in crank shafts, u.S. Patent No. 1085443 (Jan. 1914).

[9] J. P. Den Hartog, Mechanical vibrations, Mc Graw Hill, 1956. 
[10] B. F. F. B. J. Vidmar, S. W. Shaw, B. K. Geist, Nonlinear interactions in systems of multiple order centrifugal pendulum vibration absorbers, Journal of Vibration and Acoustics 135 (2013) 061012.

[11] A. Renault, O. Thomas, H. Mahé, Numerical antiresonance continuation of structural systems, Mechanical Systems and Signal Processing 116 (2019) 963-984.

[12] M. Berardengo, O. Thomas, C. Giraud-Audine, S. Manzoni, Improved shunt damping with two negative capacitances: an efficient alternative to resonant shunt, Journal of Intelligent Material Systems and Structures 28 (16) (2017) 2222-2238.

[13] J. C. Snowdon, Vibration and shock in damped mechanical systems, J. Wiley, 1968.

[14] G. Genta, Vibration dynamics and control, Springer, 2009.

[15] F. Lanchester, Damping torsional vibrations in crank-shafts, US Patent 1,085,443 (Jan. 27 1914).

URL https://www.google.com/patents/US1085443

[16] D. Charleux, G. C., F. Thouverez, J. Dupeux, Numerical and experimental study of friction damping blade attachments of rotating bladed disks, Journal of Rotating Machinery 2006 (2006) 13.

[17] D. Laxalde, F. Thouverez, Forced response analysis of integrally bladed disks with friction ring dampers, Journal of Vibration and Accoustics 132 (1) (2010) 011013-1-011013-9.

[18] J. Den Hartog, Forced vibrations with combined coulomb and viscous friction, Trans. ASME 53 (15) (1931) 107-115.

[19] J. Den Hartog, J. Ormondroyd, Torsional vibration dampers, Transactions of the American Society of Mechanical Engineers, Applied Mechanics Division 52 (1929) 133-152.

[20] S. Ye, K. A. Williams, Torsional friction damper optimization, Journal of sound and vibration 294 (3) (2006) 529-546.

[21] V. Bapat, P. Prabhu, Optimum design of lanchester damper for a viscously damped degree of freedom system by using minimum force transmissibility criterion, Journal of Sound and Vibration 67 (1) (1979) 113-119.

[22] V. Bapat, P. Prabhu, Optimum design of a lanchester damper for a viscously damped single degree of freedom system subjected to inertial excitation, Journal of Sound and Vibration 73 (1) (1980) 113-124.

[23] Z.-F. Fu, J. He, Modal analysis, Butterworth-Heinemann, 2001. 